\title{
Nursing Student Attitudes toward Euthanasia: A Cross-Sectional Study
}

\section{Kazem Hosseinzadeh}

Social Determinants of Health Research Center, Qazvin University of Medical Sciences, Qazvin, Iran

\section{Hossein Rafiei}

Student Research Center, School Of Nursing and Midwifery, Qazvin University of Medical Sciences, Qazvin, Iran

\begin{abstract}
Background: Euthanasia is among the most common and controversial end-of-life care issues. Examining the attitudes of nursing students to this issue is important because they may well encounter these issues during the course of their clinical placements.

Research aims: This study aims to examine the attitudes of a sample of Iranian nursing students towards euthanasia.

Research design: This is a descriptive cross-sectional study.

Participants and research context: Using convenience sampling, 382 Muslim nursing students were enrolled in this study.

Methods: Data were collected using a demographic variables checklist and a self-administered questionnaire that included a definition of euthanasia and II closed questions that sought to record participants' level of agreement with euthanasia based on a Likert scale.

Ethical consideration: Consent for participation was implicit, indicated by the participants having returned the completed questionnaires. Participants were assured that their data would remain anonymous, be kept confidential and be stored safely.

Findings: Of the 382 participants, $61.5 \%$ were female, and the remainder were male. The mean age was $62.6 \pm$ I4.I years (range: $32-91$ years). In total, $34.2 \%, 41.6 \%$ and $24 \%$ of students reported a negative, neutral and positive attitude to euthanasia, respectively. Most students with clinical experience, and $38.5 \%$ of students with no clinical experience, indicated their agreement with active euthanasia.

Discussion: There are a number of misconceptions among Iranian Muslim nursing students regarding the definition of euthanasia. Nonetheless, most students exhibit positive attitudes to euthanasia consistent with their clinical experiences.
\end{abstract}

Conclusion: It is recommended to explore the factors that induced nursing students' tendency to euthanasia.

\section{Keywords}

Developing countries, euthanasia, Iran, legislation, Muslim countries, nursing

Corresponding author: Kazem Hosseinzadeh, Social Determinants of Health Research Center, Qazvin University of Medical Sciences, Qazvin, Iran.

Email: khz@qums.ac.ir 